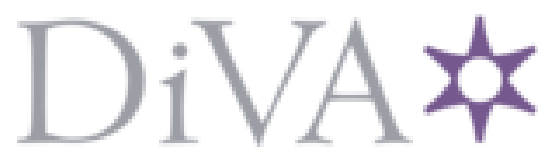

http://www.diva-portal.org

This is the published version of a paper presented at 4th International Conference, ICSI 2013.

Citation for the original published paper:

Parsapoor, m., Bilstrup, U. (2013)

Ant Colony Optimizationfor Channel Assignment Problems in Clustered Mobile Ad Hoc Network.

In: (ed.),

http://dx.doi.org/10.1007/978-3-642-38703-6_37

N.B. When citing this work, cite the original published paper.

Permanent link to this version:

http://urn.kb.se/resolve?urn=urn:nbn:se:hh:diva-23634 


\title{
Ant Colony Optimization for Channel Assignment Problem in a Clustered Mobile Ad Hoc Network
}

\author{
Mahboobeh Parsapoor and Urban Bilstrup \\ School of Information Science, Computer and Electrical Engineering \\ Halmstad University \\ \{mahboobeh.parsapoor, urban.bilstrup\} @hh.se
}

\begin{abstract}
This paper presents an ant colony optimization (ACO) method as a method for channel assignment in a mobile ad hoc network (MANET), where achieving high spectral efficiency necessitates an efficient channel assignment. The suggested algorithm is intended for graph-coloring problems and it is specifically tweaked to the channel assignment problem in MANET with a clustered network topology. A multi-objective function is designed to make a tradeoff between maximizing spectral utilization and minimizing interference. We compare the convergence behavior and performance of ACO-based method with obtained results from a grouping genetic algorithm (GGA).
\end{abstract}

Keywords: Ant colony optimization, Channel assignment problem, Co-channel Interference, Spectral efficiency.

\section{Introduction}

Ant colony optimization (ACO) is a type of meta-heuristic algorithm that has been widely used in wireless communication, in particular ad hoc networks. So far, several ACO-based routing algorithms (e.g., ANT_AODV, POSANT) [1], [2] have been proposed to provide efficient routing methods for MANETs. ACO has also been proposed as a basis for clustering algorithms. The ACO-based clustering algorithms have shown the capability to provide a scalable and stable clustered network structure [3], [4]. Using ACO-based channel assignment schemes in cellular networks have also been studied. In wireless communication, finding an optimal channel assignment has been proven as an NP-hard problem [5], [6]. This means that an optimal channel assignment scheme could not be found in the polynomial time by using the traditional exhaustive search methods e.g. branch and bound. In contrast, meta-heuristic methods, e.g. genetic algorithms (GA), swarm intelligence (SI) and in particular ant colony optimization (ACO), can be used to find near optimal solutions in polynomial time [5]-[8]. A channel assignment scheme in a clustered MANET attempts to assign a minimum number of channels to the clusters considering an interference constraint. For this problem, meta-heuristic methods, e.g. grouping genetic algorithm (GGA) and ACO, could provide near optimal solutions. They can converge towards the Pareto front for maximizing spectral efficiency and minimizing the co-channel interference between cluster heads. The rest of this paper is organized as follows: in Section 2, 
related works in applying ACO on MANETs are briefly reviewed. We also mention related studies in channel assignment problems. Section 3 suggests the use of ACO for channel assignment and illustrates how ACO can be applied as a channel assignment scheme. Section 4 presents the results of the suggested methods for channel assignment in several different scenarios and provides a comparison with another heuristic method. Finally, the paper is concluded with some finale notes in Section 5 .

\section{Related Studies in Channel Assignment Problem}

ACO-based methods have been widely used to provide efficient routing algorithms. They can minimize the delay in routing and control communication overheads [1], [2]. Numerous cluster formation algorithms have also developed combining ACO based algorithms with the traditional clustering algorithms, such as weighted clustering algorithms [5]-[8]. The channel assignment problem was early defined as a frequency assignment problem in cellular communication systems [10]-[13]. However, it is not only a problem of cellular communication systems, it exists in all kinds of wireless networks, e.g., wireless local area network (WLAN), wireless meshes network (WMN), mobile ad hoc network (MANET) and cognitive radio network (CRN). They all require an efficient channel assignment scheme to address scalability, stability, throughput, connectivity, routing, and fault tolerance [10]-[13]. Generally speaking, the channel assignment problem can be defined as finding a desirable scheme to minimize the number of channels needed for maximizing spatial reuse and at the same time satisfying interference constraints (i.e. a co-channel interference constraint). A clustered MANET is partitioned into groups of mobile nodes to provide a well-organized scalable structure for routing algorithms, power control mechanisms and spectrum management methods [10]. A common structure for a clustered network topology is based on defining three types of nodes: cluster head, gateway and ordinary nodes. The cluster head, the master of a cluster, is responsible for allocating resources and coordinates the intra cluster communication. The gateway, which is a common node between two or more clusters, provides the connectivity between clusters. Other nodes are ordinary nodes that determine the boundary of clusters, which depends on the transmission range and the node density [14]. In such a network topology, the channel assignment problem can be defined as finding a desirable scheme for assigning orthogonal channels (time, frequency or code) to the cluster heads [10]. So far, several heuristic methods e.g., greedy algorithms and genetic algorithms have been applied to solve this problem, which has been referred to as a cluster based coloring algorithm [15].

\section{Ant Colony Optimization for Cluster- Based MANETs}

Ant colony optimization meta-heuristic (ACO_MH) is a collection of algorithms which are inspired by the 'foraging behavior of real ants' [14]. Real ants start from the nest and use both local and global knowledge to construct the shortest path to the 
source of food. The ACO-based algorithms imitate this behavior to solve optimization problems. For an ACO-based algorithm, the problem is represented by a graph, $G=(V, E)$ where, the nodes, $V$, represent the components of the problem and the links, $E$, show the transition paths (i.e., partial parts of the solution) between the nodes. An optimal solution is a sequence of nodes with minimum cost function. The general components of the ACO-based algorithm are summarized as follows [16][17]: 1) A graph that represents the optimization problem. 2) A population of ants, $N_{a n t}=\left\{a n t_{1}, \ldots, a n t_{n_{a n t}}\right\}$, where $n_{a n t}$ determines the number of ants that traverse the graph; they memorize the traversed paths. 3) A set of feasible nodes, in order to avoid forming a loop during the path construction. This set is represented as $N_{i}^{k}$; it determines the feasible nodes from the perspective of ant $k$ when it is placed on $i^{\text {th }}$ node. 4) Initial states which are assigned to ants and determine the source nodes for the ants (each ant can start from different nodes). 5) A 'probabilistic transition rule' [16] is used by each ant to make a decision to move to the next node. It is defined on the basis of the heuristic information and the pheromone intensity. 6) A Heuristic function, which is a 'problem dependent function' [16], to indicate the desirability of the selected node. 7) Pheromone intensity that represents the desirability of the selected path (i.e., the path between the current node and the next node) from the perspective of other ants. 8) A cost function that is assigned to each ant that constructs a complete path from the source node to destination node. The cost function is defined on the basis of the optimization problem and can be utilized to evaluate the performance of that ant. 9) An updating rule for pheromone intensity that is used to determine the effect of the previous deposited pheromones which is defined on the basis of the cost function.

\subsection{An ACO-Based Method for Channel Assignment Problem}

To solve the channel assignment problem using ACO, a graph of the problem is represented as $G^{\prime \prime}=\left(V^{\prime \prime}, E^{\prime \prime}\right)$, where $V^{\prime \prime}$ represents the cluster heads and $E^{\prime \prime}$ represents the adjacency between the clusters, i.e. they have common nodes with each other. Thus, the number of nodes in $G^{\prime \prime}$ determines the number of clusters, $N_{c}$.

The steps of this algorithm are similar to the steps of the ACO-based graph coloring algorithm, i.e. channels are equivalent with colors. At initialization state, ants are placed on the nodes of $G^{\prime \prime}$; they are preferably placed on nodes that have more adjacent nodes, i.e. higher degree. This parameter, $N_{\text {nei, } i}$, calculates the number of adjacent nodes of node $i$. Then, each ant is assigned a set of feasible channels, $N_{c h}^{k}=\left\{c h_{1}, \ldots, c h_{n_{c h}^{k}}\right\}$, that is randomly chosen from the available set of channels, $N_{\text {Avaialble_channel }}=\left\{c h_{1}, \ldots, c h_{n_{c h}}\right\}$. The parameters $n_{c h}^{k}$ and $n_{c h}$ determine the 
number of available channels for $k^{\text {th }}$ ant and the number of available channels for the problem. The set of feasible channels is updated when an ant selects a specific channel for the current node. We define two probabilistic transition rules. The first probabilistic transition rule is defined for choosing the next node $p_{i j}^{\prime k}$; it is the probability of choosing node $j$ by the $k^{\text {th }}$ ant; while it placed at node $i$. It is defined on the basis of the heuristic information, $\eta_{i j}^{\prime}(t)$ and pheromone intensity, $\tau_{i j}^{\prime}(t)$. In the equation (1), $N_{i}^{k}$ represents the sets of feasible nodes from the perspective of the $k^{\text {th }}$ ant at node $i$. Two parameters, $\alpha$ and $\beta$ determine the influence of pheromone concentration and heuristic information respectively.

$$
p_{i j}^{\prime k}(t)=\frac{\tau_{i j}^{\prime \alpha}(t) \eta_{i j}^{\prime \beta}(t)}{\sum_{j \in N_{i}^{k}(t)} \tau_{i j}^{\prime \alpha}(t) \eta_{i j}^{\prime \beta}(t)}
$$

The second probabilistic transition rule, $p_{i c}^{\prime \prime k}$ is defined for choosing a channel for the current node. The equation (2) defines the probabilistic function using the heuristic information, $\eta_{i c}^{\prime \prime}(t)$ and pheromone intensity, $\tau_{i c}^{\prime \prime}(t)$. In this equation, $N_{c h, i}{ }^{k}$ shows the set of feasible colors from the perspective of the $k^{\text {th }}$ ant at node $i$.

$$
p_{i c}^{\prime \prime k}(t)=\frac{\tau_{i c}^{\prime \prime \alpha}(t) \eta_{i c}^{\prime \prime \beta}(t)}{\sum_{j \in N_{c h, i}^{k}(t)} \tau_{i c}^{\prime \prime \alpha}(t) \eta_{i c}^{\prime \prime \beta}(t)}
$$

Ants construct the solution by incrementally choosing one node and assign one color to that node. The completed path is a sequence of nodes along their assigned colors while satisfying the constraint that two adjacent nodes should be assigned different colors. The cost function is calculated on the basis of the traversed paths and assigned colors. Using the probabilistic transition rules as equations (1) and (2), ants choose the next node and assign a channel to that node. The ants traverse the graph and assign channels to the nodes of the graph satisfying the co-channel interference requirement.

The two heuristic functions are defined as equations (3) and (4), respectively. The heuristic function $\eta_{i j}^{\prime}$ is defined for choosing nodes and is determined by the equation (3). When an ant is at node $i$, the heuristic value for choosing the next node $j$ is calculated according to equation (3). The parameter $N_{\text {unallocated, } i}^{k}$ calculates the set of neighbors of node $i$ that have not been allocated channels from the perspective of $k^{\text {th }}$ ant. 


$$
\eta_{i j}^{\prime}=\frac{1+\left|N_{\text {unallocated }, i}^{k}\right|}{1+\left|N_{\text {nei, } i}\right|}
$$

The equation (4) defines the heuristic function $\eta_{i j}^{\prime \prime}(t)$ that is used for choosing the channel for the current node. The equation (4) calculates the desirability of choosing a channel, $c h_{j}$, when the ant is placed on node $i$.

$$
\eta_{i c h}^{\prime \prime}(t)=\frac{1+n_{\text {diff }-c h}}{1+n_{\text {simi-ch }}}
$$

The parameter $n_{d i f f-c h}$ is calculated as the maximum number of different channels for the set $\left\{c h_{j}, N_{c h, i}{ }^{k}\right\}$. The parameter $n_{s i m i-c h}^{k}$ calculates the maximum number of similar channels for the $\operatorname{set}\left\{c h_{j}, N_{\text {Assigned_channel, } i}^{k}\right\}$, where, $N_{\text {Assigned_channel, } i}^{k}$ is the assigned channels to the neighbors of $i$ from the perspective of $k^{\text {th }}$ ant.

As mentioned in the previous section, two rules are utilized to update the pheromone. The first rule is utilized to choose a node and the second rule is used for choosing a channel (i.e., color). The updating rules of nodes are defined according equation (5). The parameter, $F_{i j}^{\text {Best }}$, represents the cost function of the globally best ant, Best, for the path between node $i$ and node $j$. The parameter $N_{i}^{\text {Best }}$ represents the set of feasible nodes from the perspective of the globally best ant at node $i$.

$$
\Delta \tau_{i, j}^{\prime}=\frac{F_{i j}^{\text {Best }}}{\left|N_{i}^{\text {Best }}\right|}
$$

Another updating rule is defined as equation (6). The parameter $n_{\text {simi-ch }}^{\text {Best }}$ is the number of similar channels in $\left\{c h_{j}, N_{\text {Assigned_channel, } i}^{\text {Best }}\right\}$.

$$
\Delta \tau_{i j}^{\prime \prime}=\frac{n_{\text {simi-ch }}^{\text {Best }}}{\left|N_{i}^{\text {Best }}\right|}
$$

Assuming a channel assignment scheme by ant $k_{\text {is determined as }} \mathbf{x}^{k}$; it is an

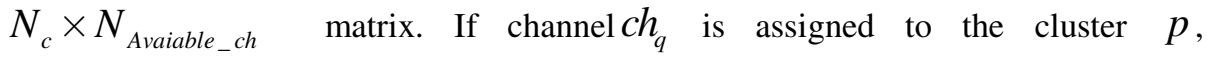
$x_{p q}=1$ otherwise it is 0 . The optimization function can be formulated according to equation (7), where, $S_{\text {simi-ch }}$ is defined as (8) and, $N_{C H}$ is the number of cluster heads. 


$$
\begin{gathered}
\arg \max \sum_{p=1}^{N_{\text {Avaiable_ch }}} \sum_{q=1}^{N_{c}} x_{p q}+S_{\text {simi_ch }} \\
S_{\text {simi-ch }}=\max _{p=1}^{N_{\text {Avaiable_ch }}}\left\{\sum_{q=1}^{N_{c}} x_{p q}\right\}
\end{gathered}
$$

\section{Simulation}

\subsection{Simulation Model}

For implementing simple scenarios of MANETs, we use a discreet even simulation Implemented in MATLAB. The main goal of this channel assignment algorithm is to assign logical channels (defined as orthogonal frequency hopping sequences) to the clusters for intra-cluster scheduling. The main assumptions of our simulated models are described as follows: 1) The suggested algorithm is implemented for a snapshot of a MANET; thus, there is no change in the network topology during the procedure to select and assign channels to the clusters. 2) The nodes are placed in an area with a $1000 \times 1000$ meter square and the position of each individual node has two coordinates, $\boldsymbol{x}$ and $\boldsymbol{y}$, that are drawn from a uniform distribution [0, 1000]. The mobility and traffic of nodes are ignored. 3) All of the nodes are assumed to have omni directional antenna with a similar transmission power. 4) The channel model and interference model are considered as free-space path loss models and disk graph models respectively. The interference range is assumed two times to the transmission range. 5) The nodes are clustered using Lowest ID (LID). 6) There is a centralized controller e.g., base station that senses the available channels; it decides for an ondemand channel assignment scheme on the basis of the unassigned channels; thus, it avoids co-channel interference with previous assigned channels.

\subsection{Simulation Results}

First, we consider the MANETs that consist of 75 nodes. The MANETs are different in transmission ranges (i.e., $\mathrm{TR}=100,200$ and 300 meters), transmission powers (i.e., 2, 4 and 6 mill watt) and the number of clusters 31,13 and 3 respectively. The number of allocated channels using ACO and GA is depicted in Figure 1. It becomes clear that the number of required channels is dependent upon the number of clusters and the topology of the network. However, for the same topology, the number of assigned channels is smaller for ACO than GGA. (See Figure 4 the black and grey bars). It can also be observed that the number of assigned channels do not significantly change when the number of clusters increases. Thus, this method would be scalable for a large sized MANET. Figure 2 presents the obtained interference power using an ACO-based scheme and GA-based scheme. In the MANET with 31 clusters (i.e., $\mathrm{TR}=100$ ), the ACO-based method has smaller value of interference power in comparison with the GA-based method. Using GA-based method in 
MANET with a small number of clusters, the interference power becomes very low. In this case, using a GA-based channel assignment scheme, each cluster has been assigned a different channel. As the second test, the convergence behavior of the ACO algorithm is evaluated for the MANET with 31 clusters (the scenario is similar to the previous scenario, here we focus on the number of iterations to achieve a near optimal solution). We compare the results of the ACO with GGA during 200 iterations; while the size of population is considered as 15. Figure 3 depicts the average and minimum values of the objective function. It shows that the ACO-based method converges after approximately 10 iterations, while the GGA converges after 120 iterations. It is also noticeable that the average and minimum values of objective functions using the GGA differ to a large extent and it needs a greater number of iterations to converge to the global minimum.

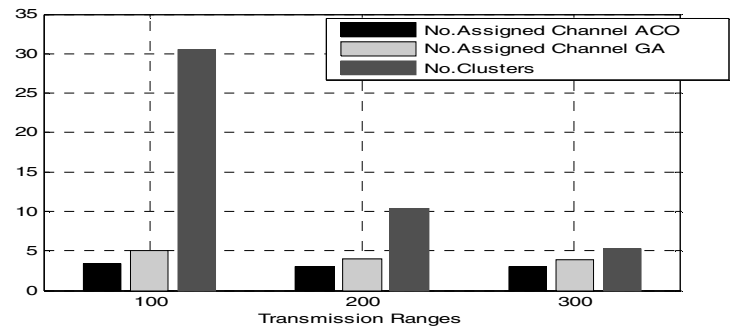

Fig. 1. The average number of assigned channels and clusters for a network with 75 nodes

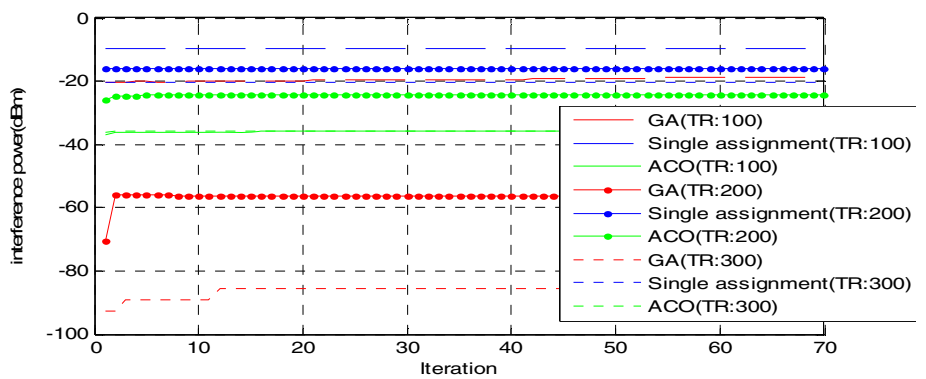

Fig. 2. Demonstrates the interference power between cluster heads for different MANETs

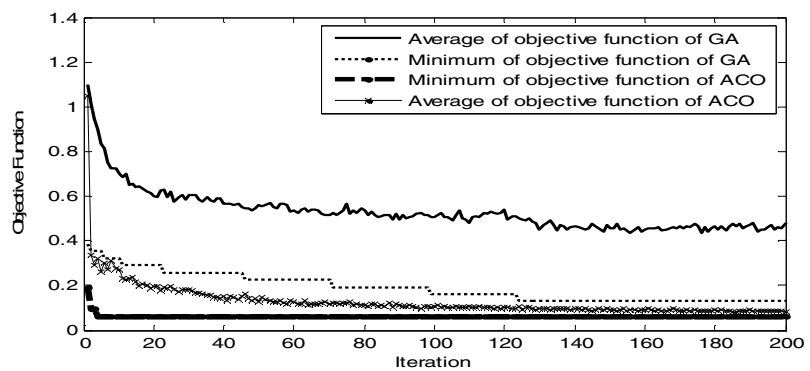

Fig. 3. The minimum and average of objective functions for a network with 30 clusters 


\section{Conclusion}

In this paper, we first develop an ACO-based method for the standard graph coloring problem; then it is extended for the channel assignment problem. We evaluated this method in the MANETs with different numbers of clusters. The results have verified that the proposed ACO algorithm has the capability to find a scheme with a minimum number of assigned channels. The results have also indicated that the ACO-based method provides a stable and scalable solution; the performance of an ACO-based channel allocation scheme does not seem to be dependent on the size of MANETs (e.g., the number of clusters in MANETs). Developing an ACO-based distributed scheme has been considered as future works. We will also replace the lowest ID clustering algorithm with an ACO-based clustering method to effectively address the channel assignment problem.

\section{References}

1. Kamali, S., Opatrny, J.: POSANT: A Position Based Ant Colony Routing Algorithm for Mobile Ad-hoc Networks. In: ICWMC 2007 (2007)

2. Jyoti Jain, R.G., Bandhopadhyay, T.K.: Ant Colony Algorithm in MANET-Local link repairing of AODV. In: ICECT (2011)

3. Di Caro, G.A., Ducatelle, F., Gambardella, L.M.: AntHocNet: An ant-based hybrid routing algorithm for mobile ad hoc networks. In: Yao, X., et al. (eds.) PPSN 2004. LNCS, vol. 3242, pp. 461-470. Springer, Heidelberg (2004)

4. Shayeb, I.G., Hussein, A.H., Nasoura, A.B.: A Survey of Clustering Schemes for Mobile Ad-Hoc Network (MANET). American Journal of Scientific Research (2), 135-151 (2011)

5. Kumar, V., Balasubramanie, P.: Ant Colony Optimization Using Hierarchical Clustering in Mobile Ad Hoc Networks. Journal of Scientific Research (4), 549-560 (2011)

6. Yu, J.Y., Chong, P.H.J.: A survey of clustering schemes for mobile ad hoc networks. IEEE Communications Surveys \& Tutorials 7, 32-48 (2005)

7. Zhang, J., Wang, B., Zhang, F.: A Distributed Approach of WCA in Ad-Hoc Network. In: Proceeding 6th International Conference on Wireless Communications, Networking and Mobile Computing, pp. 1-5 (2010)

8. Sampath, A., Tripti, C., Thampi, M.: An ACO algorithm for effective cluster head election. International Journal of Advances in Information Technology (JAIT) 2(1), 50-56 (2011)

9. Li, L., Yong, G.: Ant-colony optimization based on cluster routing protocol of Ad Hoc. In: ICCET, pp. 304-308 (2010)

10. Audhya, K., Sinha, S., Ghosh, C., Sinha, B.P.: A survey on the channel assignment problem in wireless networks. Wirel. Commun. Mob. Comput. 11, 583-609 (2010)

11. Wong, S.H.: Channel Allocation for Broadband Fixed Wireless Access Networks. PhD dissertation, University of Cambridge, Department of Engineering, UK (2003)

12. Maniezzo, V., Carbonaro, A.: An ANTS heuristic for the frequency assignment problem. Future Gener. Comp. Sy. 16, 927-935 (2000)

13. Hou, T.C., Tsai, T.J.: On the Cluster Based Dynamic Channel Assignment for Multi hop Ad Hoc Networks. J. Commun. Netw. 4(1), 40-47 (2002) 
14. Ephremedis, A., Kutten, S.: Scheduling Broadcasts in Multihop Radio Networks. IEEE Transactions on Communications COM-38, 456-460 (1990)

15. Wu, H., Zhong, Z., Hanzo, L.: A cluster-head selection and update algorithm for ad hoc networks. In: Procedding of the IEEE Globecomm Conference, pp. 1-5 (2010)

16. Engelbrecht, A.P.: Fundamentals of Computational Swarm Intelligence. John Wiley \& Sons, UK (2005)

17. Felix, T.S.C., Tiwari, M.K.: Swarm Intelligence: Focus on Ant and Particle Swarm Optimization. I-Tech Education and Publishing (2007) 